\title{
Distances between intuitionistic fuzzy sets of second type with application to diagnostic medicine
}

\author{
P. A. Ejegwa ${ }^{1}$ and I. M. Adamu ${ }^{2}$ \\ ${ }^{1}$ Department of Mathematics/Statistics/Computer Science, University of Agriculture \\ P. M. B. 2373, Makurdi, Nigeria \\ e-mails: ejegwa. augustine@uam.edu.ng, ocholohi@gmail.com \\ ${ }^{2}$ Department of Mathematics, Federal University Dutse \\ P. M. B. 7156, Dutse, Nigeria \\ e-mail: idreesmuhammadadam@gmail.com
}

Received: 18 July 2019

Revised: 20 September 2019

Accepted: 10 October 2019

\begin{abstract}
The concept of intuitionistic fuzzy sets of second type (IFSST) generalizes intuitionistic fuzzy sets (IFS) and thus, has many applications in decision making problems. The main feature of IFSST is that it is characterized by three parameters, namely: membership degree, non-membership degree and degree of indeterminacy in such a way that the sum of the square of each of the parameters is one. The purpose of this paper is to present the axiomatic definition of distance between IFSST, taking into account the three parameters that describe the sets and to investigate numerically, the validity of some distances between intuitionistic fuzzy sets introduced by E. Szmidt and J. Kacprzyk in IFSST environment. Finally, we explore the application of IFSST in diagnostic medicine by employing normalized Hamming distance of IFSST to calculate the distance between patients and diseases, because it provides a reliable distance with respect to other distances. Actually, by using the distance between patients and diseases (both in IFSST values), with recourse to the corresponding symptoms observe in the patients and of the diseases, we determine the illness of the paients. These distances are suggestible to be deployed in solving multicriteria decision making problems.
\end{abstract}

Keywords: Diagnostic medicine, Distance measure, Fuzzy set, Intuitionistic fuzzy set, Intuitionistic fuzzy set of second type.

2010 Mathematics Subject Classification: 20N20, $03 E 72$. 


\section{Introduction}

Zadeh [24] introduced the idea of fuzzy set which has a membership function, $\mu$, that assigns to each element of the universe of discourse, a number from the unit interval $[0,1]$ to indicate the degree of belongingness to the set under consideration. Fuzzy set was generalized from classical sets theory by allowing intermediate situations between the whole and nothing. In a fuzzy set, a membership function which replaces the characteristic function in crisp sets, is defined to describe the degree of membership of an element to a class. Fuzzy set which is the extension of crisp set provides a means of representing and handling vagueness and imperfectly described knowledge. Notwithstanding, a fuzzy set cannot model vagueness precisely because there is no means to attribute reliability information to the membership degrees. The conspicuous presence of vagueness/imprecision in life necessitated researchers to develop some mathematical frameworks that can cope vagueness/imprecision more accurately than fuzzy sets [12].

Out of the several mathematical frameworks which generalize fuzzy sets, the concept of intuitionistic fuzzy sets (IFS) introduced by Atanassov [1] is interesting and resourceful. IFS incorporates both a membership function, $\mu$, and a non-membership function, $\nu$, with a hesitation margin, $1-\mu-\nu=\pi$. Elaborate exploration on IFS has been carried out, see [2,4-6]. The idea of IFS seems to be resourceful in modelling many real life situations like negotiation processes, psychological investigations, reasoning, medical diagnosis, career determination, among others [7-13,17-22].

There are cases where $\mu+\nu \geq 1$ unlike in IFS (where $\mu+\nu \leq 1$ ). This limitation in IFS naturally led to the generalization of IFS as intuitionistic fuzzy sets of second type (IFSST) proposed by Atanassov [3]. An identical set called Pythagorean fuzzy set has been studied in literature [23] without a recourse to IFSST. In fact, Pythagorean fuzzy set is the same as IFSST. We feel that the development of Pythagorean fuzzy set theory independent of IFSST does not augur well since IFSST was proposed in 1989 (twenty four years before Pythagorean fuzzy set).

IFSST is a reliable tool to deal with vagueness considering the membership grade, $\mu$ and the non-membership grade, $\nu$ satisfying the condition $\mu+\nu \geq 1$. As a generalized set, IFSST has close relationship with IFS. This construct can be used to characterize uncertain information more sufficiently and accurately than IFS. Some operations on IFSST have been studied [14].

The notion of distances between IFS $[15,16]$, which is very applicable in real-life situations, is the compelling motivation of this paper. In this paper, we extend the idea of distances between IFS $[15,16]$ to IFSST and explore their application in medical diagnosis to determine the illness suffered by patients. We deploy the normalized Hamming distance for the application because it is the most reliable of the distance measures proposed in IFSST, after a reliability test is conducted. The paper is organized as follows: Section 2 provides some preliminaries on fuzzy sets, IFS as foundations to the idea of IFSST, while Section 3 covers the notion of IFSST and some distance measures between them with their numerical verifications. We present the application of IFSST to diagnostic medicine in Section 4. Finally, Section 5 concludes the paper and provides direction for future studies. 


\section{Preliminaries}

We recall some basic notions of fuzzy sets and intuitionistic fuzzy sets.

\subsection{Fuzzy sets}

Definition 2.1 ([24]). Let $X$ be a nonempty set. A fuzzy set $A$ of $X$ is characterized by a membership function $\mu_{A}: X \rightarrow[0,1]$, where

$$
\mu_{A}(x)= \begin{cases}1, & \text { if } x \in X \\ 0, & \text { if } x \notin X \\ (0,1) & \text { if } x \text { is partly in } X\end{cases}
$$

Alternatively, a fuzzy set $A$ of $X$ is an object having the form

$$
A=\left\{\left\langle x, \mu_{A}(x)\right\rangle \mid x \in X\right\} \text { or } A=\left\{\left\langle\frac{\mu_{A}(x)}{x}\right\rangle \mid x \in X\right\},
$$

where the function

$$
\mu_{A}(x): X \rightarrow[0,1]
$$

defines the degree of membership of the element, $x \in X$.

The closer the membership value $\mu_{A}(x)$ to 1 , the more $x$ belongs to $A$, where the grades 1 and 0 represent full membership and full non-membership. In classical set theory, the membership of elements to a set is assessed in binary terms according to a bivalent condition; an element either belongs or does not belong to the set. Classical bivalent sets are called in fuzzy set theory crisp sets.

Let us consider two examples;

(i) All employees of $X Y Z$ who are over $1.8 \mathrm{~m}$ in height.

(ii) All employees of $X Y Z$ who are tall.

The first example is a classical set with a universe (all $X Y Z$ employees) and a membership rule that divides the universe into members (those over $1.8 \mathrm{~m}$ ) and non-members. The second example is a fuzzy set because some employees are definitely in the set and some are definitely not in the set, but some are borderline.

This distinction between the ins, the outs and the borderline is made more exact by the membership function, $\mu$. If we return to our second example and let $A$ represent the fuzzy set of all tall employees and $x$ represent a member of the universe $X$ (i.e. all employees), then $\mu_{A}(x)$ would be $\mu_{A}(x)=1$ if $x$ is definitely tall or $\mu_{A}(x)=0$ if $x$ is definitely not tall or $0<\mu_{A}(x)<1$ for borderline cases. 


\subsection{Intuitionistic fuzzy sets}

Definition 2.2 ([1,2]). Let a nonempty set $X$ be fixed. An IFS $A$ of $X$ is an object having the form

$$
A=\left\{\left\langle x, \mu_{A}(x), \nu_{A}(x)\right\rangle \mid x \in X\right\}
$$

or

$$
A=\left\{\left\langle\frac{\mu_{A}(x), \nu_{A}(x)}{x}\right\rangle \mid x \in X\right\},
$$

where the functions

$$
\mu_{A}(x): X \rightarrow[0,1] \text { and } \nu_{A}(x): X \rightarrow[0,1]
$$

define the degree of membership and the degree of non-membership, respectively, of the element $x \in X$ to $A$, which is a subset of $X$, and for every $x \in X$,

$$
0 \leq \mu_{A}(x)+\nu_{A}(x) \leq 1
$$

For each $A$ in $X$,

$$
\pi_{A}(x)=1-\mu_{A}(x)-\nu_{A}(x)
$$

is the intuitionistic fuzzy set index or hesitation margin of $x$ in $X$. The hesitation margin $\pi_{A}(x)$ is the degree of indeterminacy of the belonging of $x \in X$, to the set $A$ and $\pi_{A}(x) \in[0,1]$. The hesitation margin is the function that expresses lack of knowledge of whether $x \in X$ or $x \notin X$. Thus,

$$
\mu_{A}(x)+\nu_{A}(x)+\pi_{A}(x)=1 .
$$

Example 2.3. Let $X=\{x, y, z\}$ be a fixed universe of discourse and

$$
A=\left\{\left\langle\frac{0.6,0.1}{x}\right\rangle,\left\langle\frac{0.8,0.1}{y}\right\rangle,\left\langle\frac{0.5,0.3}{z}\right\rangle\right\}
$$

be an intuitionistic fuzzy set of $X$. The hesitation margins of the elements $x, y, z$ to $A$ are

$$
\pi_{A}(x)=0.3, \pi_{A}(y)=0.1 \text { and } \pi_{A}(z)=0.2 .
$$

\section{Intuitionistic fuzzy sets of second type}

In this section, we succintly discuss some fundamentals of IFSST.

\subsection{Concept of intuitionistic fuzzy sets of second type}

Definition 3.1 $([3,14])$. Let $X$ be a universal set. Then, an IFSST $A$ of $X$ is a set of ordered pairs defined by

$$
A=\left\{\left\langle x, \mu_{A}(x), \nu_{A}(x)\right\rangle \mid x \in X\right\}
$$

or

$$
A=\left\{\left\langle\frac{\mu_{A}(x), \nu_{A}(x)}{x}\right\rangle \mid x \in X\right\},
$$


where the functions

$$
\mu_{A}(x): X \rightarrow[0,1] \text { and } \nu_{A}(x): X \rightarrow[0,1]
$$

define the degree of membership and the degree of non-membership, respectively, of the element $x \in X$ to $A$, which is a subset of $X$, and for every $x \in X$,

$$
0 \leq\left(\mu_{A}(x)\right)^{2}+\left(\nu_{A}(x)\right)^{2} \leq 1 .
$$

Supposing $\left(\mu_{A}(x)\right)^{2}+\left(\nu_{A}(x)\right)^{2} \leq 1$, then there is a degree of indeterminacy of $x \in X$ to $A$ defined by $\pi_{A}(x)=\sqrt{1-\left[\left(\mu_{A}(x)\right)^{2}+\left(\nu_{A}(x)\right)^{2}\right]}$ and $\pi_{A}(x) \in[0,1]$. In what follows, $\left(\mu_{A}(x)\right)^{2}+\left(\nu_{A}(x)\right)^{2}+\left(\pi_{A}(x)\right)^{2}=1$. Otherwise, $\pi_{A}(x)=0$ whenever $\left(\mu_{A}(x)\right)^{2}+\left(\nu_{A}(x)\right)^{2}=1$. We denote the set of all IFSST of $X$ by $\operatorname{IFSST}(X)$.

Example 3.2. Let $A \in \operatorname{IFSST}(X)$. Suppose $\mu_{A}(x)=0.7$ and $\nu_{A}(x)=0.5$ for $X=\{x\}$. Clearly, $0.7+0.5 \not \leq 1$, but $0.7^{2}+0.5^{2} \leq 1$. Thus $\pi_{A}(x)=0.5099$, and hence $\left(\mu_{A}(x)\right)^{2}+\left(\nu_{A}(x)\right)^{2}+$ $\left(\pi_{A}(x)\right)^{2}=1$.

Table 1 explains the difference between IFSST and IFS.

\begin{tabular}{|l|l|}
\hline IFS & IFSST \\
\hline \hline$\mu+\nu \leq 1$ & $\mu+\nu \leq 1$ or $\mu+\nu \geq 1$ \\
\hline $0 \leq \mu+\nu \leq 1$ & $0 \leq \mu^{2}+\nu^{2} \leq 1$ \\
\hline$\pi=1-(\mu+\nu)$ & $\pi=\sqrt{1-\left[\mu^{2}+\nu^{2}\right]}$ \\
\hline$\mu+\nu+\pi=1$ & $\mu^{2}+\nu^{2}+\pi^{2}=1$ \\
\hline
\end{tabular}

Table 1. Difference between IFS and IFSST

Definition 3.3. Let $A, B \in \operatorname{IFSST}(X)$. Then $A=B \Leftrightarrow \mu_{A}(x)=\mu_{B}(x)$ and $\nu_{A}(x)=\nu_{B}(x)$ $\forall x \in X$, and $A \subseteq B \Leftrightarrow \mu_{A}(x) \leq \mu_{B}(x)$ and $\nu_{A}(x) \geq \nu_{B}(x)$ (or $\left.\nu_{A}(x) \leq \nu_{B}(x)\right) \forall x \in X$. We say $A \subset B \Leftrightarrow A \subseteq B$ and $A \neq B$.

Definition 3.4. Let $A, B \in \operatorname{IFSST}(X)$. Then $A$ and $B$ are comparable to each other if $A \subseteq B$ and $B \subseteq A$.

Definition 3.5 ([3]). Let $A \in \operatorname{IFSST}(X)$. Then, the complement of $A$ denoted by $A^{c}$ is defined as

$$
A^{c}=\left\{\left\langle x, \nu_{A}(x), \mu_{A}(x)\right\rangle \mid x \in X\right\} .
$$

Remark 3.6. It is noticed that $\left(A^{c}\right)^{c}=A$. This shows the validity of complementary law in IFSST.

Definition 3.7 ([3]). Let $A, B \in \operatorname{IFSST}(X)$. Then, the following define union and intersection of $A$ and $B$ :

(i) $A \cup B=\left\{\left\langle x, \max \left(\mu_{A}(x), \mu_{B}(x)\right), \min \left(\nu_{A}(x), \nu_{B}(x)\right)\right\rangle \mid x \in X\right\}$.

(ii) $A \cap B=\left\{\left\langle x, \min \left(\mu_{A}(x), \mu_{B}(x)\right), \max \left(\nu_{A}(x), \nu_{B}(x)\right)\right\rangle \mid x \in X\right\}$. 
Definition 3.8. Let $A, B \in \operatorname{IFSST}(X)$. Then, the sum of $A$ and $B$ is defined as

$$
A \oplus B=\left\{\left\langle x, \sqrt{\left(\mu_{A}(x)\right)^{2}+\left(\mu_{B}(x)\right)^{2}-\left(\mu_{A}(x)\right)^{2}\left(\mu_{B}(x)\right)^{2}}, \nu_{A}(x) \nu_{B}(x)\right\rangle \mid x \in X\right\},
$$

and the product of $A$ and $B$ is defined as

$$
A \otimes B=\left\{\left\langle x, \mu_{A}(x) \mu_{B}(x), \sqrt{\left(\nu_{A}(x)\right)^{2}+\left(\nu_{B}(x)\right)^{2}-\left(\nu_{A}(x)\right)^{2}\left(\nu_{B}(x)\right)^{2}}\right\rangle \mid x \in X\right\} .
$$

Remark 3.9. Let $A, B, C \in \operatorname{IFSST}(X)$. By Definitions 3.7 and 3.8, the following properties hold:

(i) Complementary property;

$$
\left(A^{c}\right)^{c}=A
$$

(ii) Idempotent property;

$$
\begin{aligned}
& A \cap A=A \\
& A \cup A=A \\
& A \oplus A \neq A \\
& A \otimes A \neq A
\end{aligned}
$$

(iii) Commutative property;

$$
\begin{aligned}
& A \cap B=B \cap A \\
& A \cup B=B \cup A \\
& A \oplus B=B \oplus A \\
& A \otimes B=B \otimes A
\end{aligned}
$$

(iv) Associative property;

$$
\begin{aligned}
& A \cap(B \cap C)=(A \cap B) \cap C \\
& A \cup(B \cup C)=(A \cup B) \cup C \\
& A \oplus(B \oplus C)=(A \oplus B) \oplus C \\
& A \otimes(B \otimes C)=(A \otimes B) \otimes C
\end{aligned}
$$

(v) Distributive property;

$$
\begin{aligned}
& A \cap(B \cup C)=(A \cap B) \cup(A \cap C) \\
& A \cup(B \cap C)=(A \cup B) \cap(A \cup C) \\
& A \oplus(B \cup C)=(A \oplus B) \cup(A \oplus C) \\
& A \oplus(B \cap C)=(A \oplus B) \cap(A \oplus C) \\
& A \otimes(B \cup C)=(A \otimes B) \cup(A \otimes C) \\
& A \otimes(B \cap C)=(A \otimes B) \cap(A \otimes C)
\end{aligned}
$$


(vi) De Morgan property;

$$
\begin{aligned}
& (A \cap B)^{c}=A^{c} \cup B^{c} \\
& (A \cup B)^{c}=A^{c} \cap B^{c} \\
& (A \oplus B)^{c}=A^{c} \otimes B^{c} \\
& (A \otimes B)^{c}=A^{c} \oplus B^{c} .
\end{aligned}
$$

Definition 3.10. Let $A \in \operatorname{IFSST}(X)$. Then, the level/ground set of $A$ is defined by

$$
A_{*}=\left\{x \in X \mid \mu_{A}(x)>0, \nu_{A}(x)<1\right\} .
$$

Certainly, $A_{*}$ is a subset of $X$.

\subsection{Distances between intuitionistic fuzzy sets of second type}

First and most, we review the concept of distance measure for IFS as presented by Szmidt and Kacprzyk $[15,16]$.

Definition 3.11. Let $A, B, C \in \operatorname{IFS}(X)$. Then, the distance measure $d$ between the IFS is a function $d: I F S \times I F S \rightarrow[0,1]$ satisfying

(i) $0 \leq d(A, B) \leq 1$ (boundedness)

(ii) $d(A, B)=0$ iff $A=B$ (separability)

(iii) $d(A, B)=d(B, A)$ (symmetry)

(iv) $d(A, C)+d(B, C) \geq d(A, B)$ (triangle inequality).

For any two IFS $A$ and $B$ of $X=\left\{x_{1}, \ldots, x_{n}\right\}$, the following distance measures were proposed:

(i) Hamming distance

$$
\begin{aligned}
d_{I F S}(A, B)_{H}= & \frac{1}{2} \sum_{i=1}^{n}\left[\left|\mu_{A}\left(x_{i}\right)-\mu_{B}\left(x_{i}\right)\right|+\left|\nu_{A}\left(x_{i}\right)-\nu_{B}\left(x_{i}\right)\right|\right. \\
& \left.+\left|\pi_{A}\left(x_{i}\right)-\pi_{B}\left(x_{i}\right)\right|\right]
\end{aligned}
$$

(ii) Euclidean distance

$$
\begin{aligned}
d_{\mathrm{IFS}}(A, B)_{E}=( & \frac{1}{2} \sum_{i=1}^{n}\left[\left(\mu_{A}\left(x_{i}\right)-\mu_{B}\left(x_{i}\right)\right)^{2}+\left(\nu_{A}\left(x_{i}\right)-\nu_{B}\left(x_{i}\right)\right)^{2}\right. \\
& \left.+\left(\pi_{A}\left(x_{i}\right)-\pi_{B}\left(x_{i}\right)\right)^{2}\right)^{\frac{1}{2}}
\end{aligned}
$$


(iii) normalized Hamming distance

$$
\begin{aligned}
d_{I F S}(A, B)_{n H}= & \frac{1}{2 n} \sum_{i=1}^{n}\left[\left|\mu_{A}\left(x_{i}\right)-\mu_{B}\left(x_{i}\right)\right|+\left|\nu_{A}\left(x_{i}\right)-\nu_{B}\left(x_{i}\right)\right|\right. \\
& \left.+\left|\pi_{A}\left(x_{i}\right)-\pi_{B}\left(x_{i}\right)\right|\right]
\end{aligned}
$$

(iv) normalized Euclidean distance

$$
\begin{aligned}
d_{I F S}(A, B)_{n E}= & \left(\frac { 1 } { 2 n } \sum _ { i = 1 } ^ { n } \left[\left(\mu_{A}\left(x_{i}\right)-\mu_{B}\left(x_{i}\right)\right)^{2}+\left(\nu_{A}\left(x_{i}\right)-\nu_{B}\left(x_{i}\right)\right)^{2}\right.\right. \\
& \left.+\left(\pi_{A}\left(x_{i}\right)-\pi_{B}\left(x_{i}\right)\right)^{2}\right)^{\frac{1}{2}}
\end{aligned}
$$

for

$$
\pi_{A}\left(x_{i}\right)=1-\mu_{A}\left(x_{i}\right)-\nu_{A}\left(x_{i}\right)
$$

and

$$
\pi_{B}\left(x_{i}\right)=1-\mu_{B}\left(x_{i}\right)-\nu_{B}\left(x_{i}\right) .
$$

All these distances have been tested to for reliability with the normalized Hamming distance as the most reliable one.

Now, we give an analogous definition of distance measure for IFSST as follows:

Definition 3.12. Let $X$ be nonempty set and $A, B, C \in \operatorname{IFSST}(X)$. Then, the distance measure $d$ between the IFSST is a function

$$
d: I F S S T \times I F S S T \rightarrow[0,1]
$$

satisfying

(i) $0 \leq d(A, B) \leq 1$ (boundedness)

(ii) $d(A, B)=0$ iff $A=B$ (separability)

(iii) $d(A, B)=d(B, A)$ (symmetric)

(iv) $d(A, C)+d(B, C) \geq d(A, B)$ (triangle inequality).

Let $A$ and $B$ be IFSST of $X=\left\{x_{1}, \ldots, x_{n}\right\}$. Then, we propose the following distance measures as extension of $[15,16]$ :

(i) Hamming distance

$$
\begin{aligned}
d_{I F S S T}(A, B)_{H}= & \frac{1}{2} \sum_{i=1}^{n}\left[\left|\mu_{A}\left(x_{i}\right)-\mu_{B}\left(x_{i}\right)\right|+\left|\nu_{A}\left(x_{i}\right)-\nu_{B}\left(x_{i}\right)\right|\right. \\
& \left.+\left|\pi_{A}\left(x_{i}\right)-\pi_{B}\left(x_{i}\right)\right|\right]
\end{aligned}
$$


(ii) Euclidean distance

$$
\begin{aligned}
d_{I F S S T}(A, B)_{E}= & \left(\frac { 1 } { 2 } \sum _ { i = 1 } ^ { n } \left[\left(\mu_{A}\left(x_{i}\right)-\mu_{B}\left(x_{i}\right)\right)^{2}+\left(\nu_{A}\left(x_{i}\right)-\nu_{B}\left(x_{i}\right)\right)^{2}\right.\right. \\
& \left.+\left(\pi_{A}\left(x_{i}\right)-\pi_{B}\left(x_{i}\right)\right)^{2}\right)^{\frac{1}{2}}
\end{aligned}
$$

(iii) normalized Hamming distance

$$
\begin{aligned}
d_{I F S S T}(A, B)_{n H}= & \frac{1}{2 n} \sum_{i=1}^{n}\left[\left|\mu_{A}\left(x_{i}\right)-\mu_{B}\left(x_{i}\right)\right|+\left|\nu_{A}\left(x_{i}\right)-\nu_{B}\left(x_{i}\right)\right|\right. \\
& \left.+\left|\pi_{A}\left(x_{i}\right)-\pi_{B}\left(x_{i}\right)\right|\right]
\end{aligned}
$$

(iv) normalized Euclidean distance

$$
\begin{aligned}
d_{I F S S T}(A, B)_{n E}= & \left(\frac { 1 } { 2 n } \sum _ { i = 1 } ^ { n } \left[\left(\mu_{A}\left(x_{i}\right)-\mu_{B}\left(x_{i}\right)\right)^{2}+\left(\nu_{A}\left(x_{i}\right)-\nu_{B}\left(x_{i}\right)\right)^{2}\right.\right. \\
& \left.+\left(\pi_{A}\left(x_{i}\right)-\pi_{B}\left(x_{i}\right)\right)^{2}\right)^{\frac{1}{2}}
\end{aligned}
$$

where

$$
\pi_{A}\left(x_{i}\right)=\sqrt{1-\left[\left(\mu_{A}\left(x_{i}\right)\right)^{2}+\left(\nu_{A}\left(x_{i}\right)\right)^{2}\right]}
$$

and

$$
\pi_{B}\left(x_{i}\right)=\sqrt{1-\left[\left(\mu_{B}\left(x_{i}\right)\right)^{2}+\left(\nu_{B}\left(x_{i}\right)\right)^{2}\right]} .
$$

Proposition 3.13. Let $A, B \in \operatorname{IFSST}(X)$. Then $d(A, B)=d\left(A^{c}, B^{c}\right)$.

Proof. Straightforward from Definition 3.12

Proposition 3.14. Let $A, B, C \in \operatorname{IFSST}(X)$. Suppose $A \subseteq B \subseteq C$, then $d(A, C) \geq d(A, B)$ and $d(A, C) \geq d(B, C)$.

Proof. By Definition 3.12, the proof follows.

\subsection{Numerical verification}

We now verify whether these distance measures for IFSST satisfy the conditions in Definition 3.12. Recall that, every IFS is an IFSST but the converse is not true. Now, we evoke the following example from [16].

Example 3.15. Let us consider the following intuitionistic fuzzy sets $A$ and $B$ of $X=\{1,2,3,4$, $5,6,7\}$.

$$
\begin{gathered}
A=\left\{\left\langle\frac{0.5,0.3,0.2}{1}\right\rangle,\left\langle\frac{0.2,0.6,0.2}{2}\right\rangle,\left\langle\frac{0.3,0.2,0.5}{4}\right\rangle,\left\langle\frac{0.2,0.2,0.6}{5}\right\rangle,\left\langle\frac{1.0,0.0,0.0}{6}\right\rangle\right\} \\
B=\left\{\left\langle\frac{0.2,0.6,0.2}{1}\right\rangle,\left\langle\frac{0.3,0.2,0.5}{4}\right\rangle,\left\langle\frac{0.5,0.2,0.3}{5}\right\rangle,\left\langle\frac{0.9,0.0,0.1}{7}\right\rangle\right\} .
\end{gathered}
$$

Clearly, $A_{*} \neq B_{*}$. 
Adopting the given IFS into IFSST and adding an IFSST $C$ of $X$ to $A$ and $B$, we have

$$
A=\left\{\left\langle\frac{0.5,0.3}{1}\right\rangle,\left\langle\frac{0.2,0.6}{2}\right\rangle,\left\langle\frac{0.3,0.2}{4}\right\rangle,\left\langle\frac{0.2,0.2}{5}\right\rangle,\left\langle\frac{1.0,0.0}{6}\right\rangle\right\}
$$

where

$$
\begin{gathered}
\pi_{A}(1)=0.8124, \pi_{A}(2)=0.7746, \pi_{A}(4)=0.9327, \pi_{A}(5)=0.9592, \pi_{A}(6)=0.0000, \\
B=\left\{\left\langle\frac{0.2,0.6}{1}\right\rangle,\left\langle\frac{0.3,0.2}{4}\right\rangle,\left\langle\frac{0.5,0.2}{5}\right\rangle,\left\langle\frac{0.9,0.0}{7}\right\rangle\right\}
\end{gathered}
$$

where

$$
\begin{gathered}
\pi_{B}(1)=0.7746, \pi_{B}(4)=0.9327, \pi_{B}(5)=0.8426, \pi_{A}(7)=0.4359 \\
C=\left\{\left\langle\frac{0.4,0.5}{1}\right\rangle,\left\langle\frac{0.6,0.2}{3}\right\rangle,\left\langle\frac{0.8,0.1}{4}\right\rangle,\left\langle\frac{0.3,0.4}{5}\right\rangle,\left\langle\frac{0.7,0.2}{7}\right\rangle\right\}
\end{gathered}
$$

where

$$
\pi_{C}(1)=0.7681, \pi_{C}(3)=0.7746, \pi_{C}(4)=0.5916, \pi_{C}(5)=0.8660, \pi_{C}(7)=0.6856 .
$$

Applying the distances between IFSST, we obtain the following results:

$$
\begin{aligned}
d_{I F S S T}(A, B)_{H}= & \frac{1}{2} \sum_{i=1}^{7}[|0.5-0.2|+|0.3-0.6|+|0.8124-0.7746| \\
& +|0.2-0.0|+|0.6-1.0|+|0.7746-0.0| \\
& +|0.3-0.3|+|0.2-0.2|+|0.9327-0.9327| \\
& +|0.2-0.5|+|0.2-0.2|+|0.9592-0.8426| \\
& +|1.0-0.0|+|0.0-1.0|+|0.0-0.0| \\
& +|0.0-0.9|+|1.0-0.0|+|0.0-0.4359|] \\
= & 3.3825 \\
& \\
d_{I F S S T}(A, C)_{H}= & \frac{1}{2} \sum_{i=1}^{7}[|0.5-0.4|+|0.3-0.5|+|0.8124-0.7681| \\
& +|0.2-0.0|+|0.6-1.0|+|0.7746-0.0| \\
& +|0.0-0.6|+|1.0-0.2|+|0.0-0.7746| \\
& +|0.3-0.8|+|0.2-0.1|+|0.9327-0.5916| \\
& +|0.2-0.3|+|0.2-0.4|+|0.9592-0.8660| \\
& +|1.0-0.0|+|0.0-1.0|+|0.0-0.0| \\
& +|0.0-0.7|+|1.0-0.2|+|0.0-0.6856|] \\
= & 4.7067
\end{aligned}
$$




$$
\begin{aligned}
d_{\text {IFSST }}(B, C)_{H}= & \frac{1}{2} \sum_{i=1}^{7}[|0.2-0.4|+|0.6-0.5|+|0.7746-0.7681| \\
& +|0.0-0.6|+|1.0-0.2|+|0.0-0.7746| \\
& +|0.3-0.8|+|0.2-0.1|+|0.9327-0.5916| \\
& +|0.5-0.3|+|0.2-0.4|+|0.8426-0.8660| \\
& +|0.9-0.7|+|0.0-0.2|+|0.4359-0.6856|] \\
= & 2.2477
\end{aligned}
$$

$$
\begin{aligned}
d_{\text {IFSST }}(A, B)_{E}= & \left(\frac { 1 } { 2 } \sum _ { i = 1 } ^ { 7 } \left[(0.5-0.2)^{2}+(0.3-0.6)^{2}+(0.8124-0.7746)^{2}\right.\right. \\
& +(0.2-0.0)^{2}+(0.6-1.0)^{2}+(0.7746-0.0)^{2} \\
& +(0.3-0.3)^{2}+(0.2-0.2)^{2}+(0.9327-0.9327)^{2} \\
& +(0.2-0.5)^{2}+(0.2-0.2)^{2}+(0.9592-0.8426)^{2} \\
& +(1.0-0.0)^{2}+(0.0-1.0)^{2}+(0.0-0.0)^{2} \\
& \left.\left.+(0.0-0.9)^{2}+(1.0-0.0)^{2}+(0.0-0.4359)^{2}\right]\right)^{\frac{1}{2}} \\
= & 1.5945
\end{aligned}
$$

$$
\begin{aligned}
d_{\text {IFSST }}(A, C)_{E}= & \left(\frac { 1 } { 2 } \sum _ { i = 1 } ^ { 7 } \left[(0.5-0.4)^{2}+(0.3-0.5)^{2}+(0.8124-0.7681)^{2}\right.\right. \\
& +(0.2-0.0)^{2}+(0.6-1.0)^{2}+(0.7746-0.0)^{2} \\
& +(0.0-0.6)^{2}+(1.0-0.2)^{2}+(0.0-0.7746)^{2} \\
& +(0.3-0.8)^{2}+(0.2-0.1)^{2}+(0.9327-0.5916)^{2} \\
& +(0.2-0.3)^{2}+(0.2-0.4)^{2}+(0.9592-0.8660)^{2} \\
& +(1.0-0.0)^{2}+(0.0-1.0)^{2}+(0.0-0.0)^{2} \\
& \left.\left.+(0.0-0.7)^{2}+(1.0-0.2)^{2}+(0.0-0.6856)^{2}\right]\right)^{\frac{1}{2}} \\
= & 1.8010
\end{aligned}
$$

$$
\begin{aligned}
d_{\text {IFSST }}(B, C)_{E}= & \left(\frac { 1 } { 2 } \sum _ { i = 1 } ^ { 7 } \left[(0.2-0.4)^{2}+(0.6-0.5)^{2}+(0.7746-0.7681)^{2}\right.\right. \\
& +(0.0-0.6)^{2}+(1.0-0.2)^{2}+(0.0-0.7746)^{2} \\
& +(0.3-0.8)^{2}+(0.2-0.1)^{2}+(0.9327-0.5916)^{2} \\
& +(0.5-0.3)^{2}+(0.2-0.4)^{2}+(0.8426-0.8660)^{2} \\
& \left.\left.+(0.9-0.7)^{2}+(0.0-0.2)^{2}+(0.4359-0.6856)^{2}\right]\right)^{\frac{1}{2}} \\
= & 1.0605
\end{aligned}
$$




$$
\begin{aligned}
d_{I F S S T}(A, B)_{n H}= & \frac{1}{14} \sum_{i=1}^{7}[|0.5-0.2|+|0.3-0.6|+|0.8124-0.7746| \\
& +|0.2-0.0|+|0.6-1.0|+|0.7746-0.0| \\
& +|0.3-0.3|+|0.2-0.2|+|0.9327-0.9327| \\
& +|0.2-0.5|+|0.2-0.2|+|0.9592-0.8426| \\
& +|1.0-0.0|+|0.0-1.0|+|0.0-0.0| \\
& +|0.0-0.9|+|1.0-0.0|+|0.0-0.4359|] \\
= & 0.4832
\end{aligned}
$$

$$
\begin{aligned}
d_{\text {IFSST }}(A, C)_{n H}= & \frac{1}{14} \sum_{i=1}^{7}[|0.5-0.4|+|0.3-0.5|+|0.8124-0.7681| \\
& +|0.2-0.0|+|0.6-1.0|+|0.7746-0.0| \\
& +|0.0-0.6|+|1.0-0.2|+|0.0-0.7746| \\
& +|0.3-0.8|+|0.2-0.1|+|0.9327-0.5916| \\
& +|0.2-0.3|+|0.2-0.4|+|0.9592-0.8660| \\
& +|1.0-0.0|+|0.0-1.0|+|0.0-0.0| \\
& +|0.0-0.7|+|1.0-0.2|+|0.0-0.6856|] \\
= & 0.6724
\end{aligned}
$$

$$
\begin{aligned}
d_{\text {IFSST }}(B, C)_{n H}= & \frac{1}{14} \sum_{i=1}^{7}[|0.2-0.4|+|0.6-0.5|+|0.7746-0.7681| \\
& +|0.0-0.6|+|1.0-0.2|+|0.0-0.7746| \\
& +|0.3-0.8|+|0.2-0.1|+|0.9327-0.5916| \\
& +|0.5-0.3|+|0.2-0.4|+|0.8426-0.8660| \\
& +|0.9-0.7|+|0.0-0.2|+|0.4359-0.6856|] \\
= & 0.3211
\end{aligned}
$$

$$
\begin{aligned}
d_{I F S S T}(A, B)_{n E}= & \left(\frac { 1 } { 1 4 } \sum _ { i = 1 } ^ { 7 } \left[(0.5-0.2)^{2}+(0.3-0.6)^{2}+(0.8124-0.7746)^{2}\right.\right. \\
& +(0.2-0.0)^{2}+(0.6-1.0)^{2}+(0.7746-0.0)^{2} \\
& +(0.3-0.3)^{2}+(0.2-0.2)^{2}+(0.9327-0.9327)^{2} \\
& +(0.2-0.5)^{2}+(0.2-0.2)^{2}+(0.9592-0.8426)^{2} \\
& +(1.0-0.0)^{2}+(0.0-1.0)^{2}+(0.0-0.0)^{2} \\
& \left.\left.+(0.0-0.9)^{2}+(1.0-0.0)^{2}+(0.0-0.4359)^{2}\right]\right)^{\frac{1}{2}} \\
= & 0.6027
\end{aligned}
$$




$$
\begin{aligned}
d_{I F S S T}(A, C)_{n E}= & \left(\frac { 1 } { 1 4 } \sum _ { i = 1 } ^ { 7 } \left[(0.5-0.4)^{2}+(0.3-0.5)^{2}+(0.8124-0.7681)^{2}\right.\right. \\
& +(0.2-0.0)^{2}+(0.6-1.0)^{2}+(0.7746-0.0)^{2} \\
& +(0.0-0.6)^{2}+(1.0-0.2)^{2}+(0.0-0.7746)^{2} \\
& +(0.3-0.8)^{2}+(0.2-0.1)^{2}+(0.9327-0.5916)^{2} \\
& +(0.2-0.3)^{2}+(0.2-0.4)^{2}+(0.9592-0.8660)^{2} \\
& +(1.0-0.0)^{2}+(0.0-1.0)^{2}+(0.0-0.0)^{2} \\
& \left.\left.+(0.0-0.7)^{2}+(1.0-0.2)^{2}+(0.0-0.6856)^{2}\right]\right)^{\frac{1}{2}} \\
= & 0.6807 \\
& +(0.0-0.6)^{2}+(1.0-0.2)^{2}+(0.0-0.7746)^{2} \\
d_{I F S S T}(B, C)_{n E}= & \frac{1}{14} \sum_{i=1}^{7}\left[(0.2-0.4)^{2}+(0.6-0.5)^{2}+(0.7746-0.7681)^{2}\right. \\
& +(0.3-0.8)^{2}+(0.2-0.1)^{2}+(0.9327-0.5916)^{2} \\
& +(0.5-0.3)^{2}+(0.2-0.4)^{2}+(0.8426-0.8660)^{2} \\
& \left.\left.+(0.9-0.7)^{2}+(0.0-0.2)^{2}+(0.4359-0.6856)^{2}\right]\right)^{\frac{1}{2}} \\
= & 0.4008
\end{aligned}
$$

Table 2 gives the summary of the results.

\begin{tabular}{|l|l|l|l|l|}
\hline Distances & $\begin{array}{l}\text { Hamming } \\
\text { distance }\end{array}$ & $\begin{array}{l}\text { Euclidean } \\
\text { distance }\end{array}$ & $\begin{array}{l}n \text {-Hamming } \\
\text { distance }\end{array}$ & $\begin{array}{l}n \text {-Euclidean } \\
\text { distance }\end{array}$ \\
\hline \hline$d(A, B)$ & 3.3825 & 1.5945 & 0.4832 & 0.6027 \\
\hline$d(A, C)$ & 4.7067 & 1.8010 & 0.6724 & 0.6807 \\
\hline$d(B, C)$ & 2.2477 & 1.0605 & 0.3211 & 0.4008 \\
\hline
\end{tabular}

Table 2. Numerical outputs

\subsection{Discussion}

From Table 2, we observe that the distances between IFS (i.e., Hamming distance and Euclidean distance) $[15,16]$ do not satisfy the conditions of distance measure for IFSST as seen in Definition 3.12. However, $n$-Hamming distance and $n$-Euclidean distance satisfy the conditions of distance measure for IFSST. Thus, $n$-Hamming distance and $n$-Euclidean distance are appropriate distance measures for IFSST.

In summary; (i) $d(A, B), d(A, C), d(B, C) \in[0,1]$, for $n$-Hamming and $n$-Euclidean distances, (ii) $d(A, B)=0, d(A, C)=0$ and $d(B, C)=0$ if and only if $A=B, A=C$ and $B=C$ 
for $n$-Hamming and $n$-Euclidean distances, (iii) for $n$-Hamming and $n$-Euclidean distances, it follows that

$$
d(A, B)=d(B, A), d(A, C)=d(C, A) \text { and } d(B, C)=d(C, B)
$$

because of the use of square and absolute value, (iv) $d(A, C)+d(B, C) \geq d(A, B)$ holds for $n$-Hamming and $n$-Euclidean distances.

Notwithstanding, $n$-Hamming distance is the most reasonable/efficient of the distance measures discussed. Hence, we adopt $n$-Hamming distance for application to diagnostic medicine.

\section{Application of IFSST to diagnostic medicine}

In this section, we present an application of IFSST in diagnostic medicine. In a given pathology, suppose $S$ is a set of symptoms, $D$ is a set of diseases, and $P$ is a set of patients. Using medical knowledge of diseases and symptoms, we know that some symptoms are perculiar to a particular disease than the other, that is, each disease has perculiar symptoms which reveal a degree of association, $\mu$, and a degree of non-association, $\nu$, between the symptoms and diseases.

Now we discuss the concept of IFSST in diagnostic medicine. The methodology involves mainly three stages viz;

(i) determination of symptoms

(ii) formulation of medical knowledge based on IFSST values

(iii) determination of diagnosis using $n$-Hamming distance between $P$ and $D$.

Then, the distance, $d$ between patient and disease with respect to symptoms is given as

$$
d_{I F S S T}(P, D)_{n H}=\frac{1}{2 n} \sum_{i=1}^{n}\left[\left|\mu_{P}\left(s_{i}\right)-\mu_{D}\left(s_{i}\right)\right|+\left|\nu_{P}\left(s_{i}\right)-\nu_{D}\left(s_{i}\right)\right|+\left|\pi_{P}\left(s_{i}\right)-\pi_{D}\left(s_{i}\right)\right|\right],
$$

where $s_{i} \in S$, and $n$ is the number of symptoms.

A patient is diagnosed to suffer from a particular disease if the distance, $d$ between the disease and the patient is the least. To see the application of the method, let us make a hypothetical case.

\subsection{Experimental example}

Suppose there are four patients, $P$ given as a set $P=\{$ Ene, Ehi, Ela, Ebo $\}$ to be examined for the set of diseases, $D$ given as

$$
D=\{\text { viral fever, malaria fever, typhoid fever, stomach ulcer, chest problem }\}
$$

which have the set of symptoms, $S$

$$
S=\{\text { temperature, headache, stomach pain, cough, chest pain }\}
$$


From medical knowledge, Table 3 contains each disease and its symptoms in IFSST values.

\begin{tabular}{|l|l|l|l|l|l|}
\hline & viral fever & $\begin{array}{l}\text { malaria } \\
\text { fever }\end{array}$ & $\begin{array}{l}\text { typhoid } \\
\text { fever }\end{array}$ & $\begin{array}{l}\text { stomach } \\
\text { ulcer }\end{array}$ & $\begin{array}{l}\text { chest } \\
\text { problem }\end{array}$ \\
\hline \hline temperature & $\langle 0.4,0.0\rangle$ & $\langle 0.7,0.0\rangle$ & $\langle 0.3,0.3\rangle$ & $\langle 0.1,0.7\rangle$ & $\langle 0.1,0.8\rangle$ \\
\hline headache & $\langle 0.3,0.5\rangle$ & $\langle 0.2,0.6\rangle$ & $\langle 0.6,0.1\rangle$ & $\langle 0.2,0.4\rangle$ & $\langle 0.0,0.8\rangle$ \\
\hline stomach & $\langle 0.1,0.7\rangle$ & $\langle 0.0,0.9\rangle$ & $\langle 0.2,0.7\rangle$ & $\langle 0.8,0.0\rangle$ & $\langle 0.2,0.8\rangle$ \\
\hline cough & $\langle 0.4,0.3\rangle$ & $\langle 0.7,0.0\rangle$ & $\langle 0.2,0.6\rangle$ & $\langle 0.2,0.7\rangle$ & $\langle 0.2,0.8\rangle$ \\
\hline chest pain & $\langle 0.1,0.7\rangle$ & $\langle 0.1,0.8\rangle$ & $\langle 0.1,0.9\rangle$ & $\langle 0.2,0.7\rangle$ & $\langle 0.8,0.1\rangle$ \\
\hline
\end{tabular}

Table 3. Symptoms vs Diseases

After the samples were collected from the patients and analysed, the results contained in Table 4 are obtained.

\begin{tabular}{|l|l|l|l|l|l|}
\hline & temperature & headache & $\begin{array}{l}\text { stomach } \\
\text { pain }\end{array}$ & cough & chest pain \\
\hline \hline Ene & $\langle 0.8,0.1\rangle$ & $\langle 0.6,0.1\rangle$ & $\langle 0.2,0.8\rangle$ & $\langle 0.6,0.1\rangle$ & $\langle 0.1,0.6\rangle$ \\
\hline Ehi & $\langle 0.0,0.8\rangle$ & $\langle 0.4,0.4\rangle$ & $\langle 0.6,0.1\rangle$ & $\langle 0.1,0.7\rangle$ & $\langle 0.1,0.8\rangle$ \\
\hline Ela & $\langle 0.8,0.1\rangle$ & $\langle 0.8,0.1\rangle$ & $\langle 0.0,0.6\rangle$ & $\langle 0.2,0.7\rangle$ & $\langle 0.0,0.5\rangle$ \\
\hline Ebo & $\langle 0.6,0.1\rangle$ & $\langle 0.5,0.4\rangle$ & $\langle 0.3,0.4\rangle$ & $\langle 0.7,0.2\rangle$ & $\langle 0.3,0.4\rangle$ \\
\hline
\end{tabular}

Table 4. Patients vs Symptoms

After calculating the degree of indeterminatcy using $\pi=\sqrt{1-\left[\mu^{2}+\nu^{2}\right]}$, we compute the distance between patients and diseases using Equation 1. The computational results are contained in Table 5.

\begin{tabular}{|l|l|l|l|l|l|}
\hline$d_{\text {IFSST }}(P, D)$ & viral fever & $\begin{array}{l}\text { malaria } \\
\text { fever }\end{array}$ & $\begin{array}{l}\text { typhoid } \\
\text { fever }\end{array}$ & $\begin{array}{l}\text { stomach } \\
\text { ulcer }\end{array}$ & $\begin{array}{l}\text { chest } \\
\text { problem }\end{array}$ \\
\hline \hline Ene & 0.1817 & 0.1681 & 0.2016 & 0.3619 & 0.4017 \\
\hline Ehi & 0.2850 & 0.3664 & 0.2342 & 0.1062 & 0.3002 \\
\hline Ela & 0.2484 & 0.2980 & 0.2136 & 0.3428 & 0.3740 \\
\hline Ebo & 0.1904 & 0.2228 & 0.2678 & 0.3114 & 0.3975 \\
\hline
\end{tabular}

Table 5. Decision Table

\subsubsection{Diagnostic decision}

From Table 5, the following diagnosis are determined based on the least value of the distances between $P$ and $D$ : Ene is diagnosed of malaria fever, Ehi is diagnosed of stomach ulcer, Ela is diagnosed of typhoid fever, Ebo is diagnosed of viral fever. 


\section{Conclusion}

In this paper, we proposed an axiomatic definition of distance between intuitionistic fuzzy sets of second type. It was shown that distances between intuitionistic fuzzy sets of second type should be calculated by taking into account the three parameters that describe an intuitionistic fuzzy set of second type. Taking into account all the three parameters that describe intuitionistic fuzzy sets of second type when calculating distances ensure that the distances for intuitionistic fuzzy sets and intuitionistic fuzzy sets of second type can be easily compared. We verified the authenticity of the proposed distances between IFSST in comparison to Definition 3.12, and found that Hamming and Euclidean distances are not distance measures for IFSST. But, $n$-Hamming and $n$-Euclidean distances are appropriate distance measures for IFSST. To test the applicability of the proposed distance measures in real-life problems, a medical diagnostic problem was considered via $n$-Hamming distance, for a reliable output. The distances between IFSST studied in this work could be applied in decision making of real-life problems embedded with uncertainty.

\section{Acknowledgements}

The authors are appreciative to the Editor-in-chief Professor K. T. Atanassov for his technical comments and to the anonymous reviewers for their suggestions which improved the quality of this paper.

\section{References}

[1] Atanassov, K. T. (1983). Intuitionistic Fuzzy Sets, VII ITKR Session, Sofia, 20-23 June 1983 (Deposed in Centr. Sci.-Techn. Library of the Bulg. Acad. of Sci., 1697/84) (in Bulgarian). Reprinted: Int. J. Bioautomation, 2016, 20(S1), S1-S6 (in English).

[2] Atanassov, K. T. (1986). Intuitionistic fuzzy sets, Fuzzy Set Syst., 20, 87-96.

[3] Atanassov, K. T. (1989). Geometrical Interpretation of the Elements of the Intuitionistic Fuzzy Objects, Mathematical Foundations of Artificial Intelligence Seminar, Sofia, Preprint IM-MFAIS-1-89. Reprinted: Int J Bioautomation, 2016, 20(S1), S27-S42.

[4] Atanassov, K. T. (1994). New operations defined on intuitionistic fuzzy sets, Fuzzy Sets and Systems, 61, 137-142.

[5] Atanassov, K. T. (1999). Intuitionistic Fuzzy Sets: Theory and Applications, Physica-Verlag, Heidelberg.

[6] Atanassov, K. T. (2012). On Intuitionistic Fuzzy Sets Theory, Springer, Berlin.

[7] Davvaz, B., \& Sadrabadi, E. H. (2016). An application of intuitionistic fuzzy sets in medicine, Int. J. Biomath., 9(3), 1650037 (15 pages). 
[8] De, S. K., Biswas, R., \& Roy, A. R. (2001). An application of intuitionistic fuzzy sets in medical diagnosis, Fuzzy Set Syst., 117(2), 209-213.

[9] Ejegwa, P. A. (2015). Intuitionistic fuzzy sets approach in appointment of positions in an organization via max-min-max rule, Global J. Sci. Frontier Research: F Math. Decision Sci., 15(6), 1-6.

[10] Ejegwa, P. A., Akubo, A. J., \& Joshua, O. M. (2014). Intuitionistic fuzzzy sets in career determination, J. Info. Computing Sci., 9(4), 285-288.

[11] Ejegwa, P. A., \& Modom, E. S. (2015). Diagnosis of viral hepatitis using new distance measure of intuitionistic fuzzy sets, Intern. J. Fuzzy Mathematical Archive, 8(1), 1-7.

[12] Ejegwa, P. A., \& Onasanya, B. O. (2019). Improved intuitionistic fuzzy composite relation and its application to medical diagnostic process, Notes on Intuitionistic Fuzzy Sets, 25(1), 43-58.

[13] Ejegwa, P. A., \& Onyeke, I. C. (2018). An object oriented approach to the application of intuitionistic fuzzy sets in competency based test evaluation, Ann. Commun. Math., 1(1), $38-47$.

[14] Parvathi, R., \& Palaniappan, N. (2004). Some operations on intuitionistic fuzzy sets of second type, Notes on Intuitionistic Fuzzy Sets, 10(2), 1-19.

[15] Szmidt, E. (2014). Distances and similarities in intuitionistic fuzzy sets, Springer International Publishing, Switzerland, 2014

[16] Szmidt, E., \& Kacprzyk, J. (2000). Distances between intuitionistic fuzzy sets, Fuzzy Set Syst., 114, 505-518.

[17] Szmidt, E., \& Kacprzyk, J. (2001). Intuitionistic fuzzy sets in some medical applications, Notes on Intuitionistic Fuzzy Sets, 7(4), 58-64.

[18] Szmidt, E., \& Kacprzyk, J. (2002). An intuitionistic fuzzy set based approach to intelligent data analysis: an application to medical diagnosis. In:- Recent Advances in Intelligent Paradigms and Applications, Springer, Berlin, 57-70.

[19] Szmidt, E., \& Kacprzyk, J. (2004). Medical diagnostic reasoning using a similarity measure for intuitionistic fuzzy sets, Notes on Intuitionistic Fuzzy Sets, 10(4), 61-69.

[20] Szmidt, E., \& Kacprzyk, J. (2005). A similarity measure for intuitionistic fuzzy sets and its application in supporting medical diagnostic reasoning. In:- Lecture Notes in Artificial Intelligence, Vol. 3070, Springer, Berlin, 388-393.

[21] Todorova, L., Atanassov, K. T., Hadjitodorov, S., \& Vassilev, P. (2007). On an intuitionistic fuzzy approach for decision-making in medicine (Part 1), Int. J. Bioautomation, 6, 92-101. 
[22] Todorova, L., Atanassov, K. T., Hadjitodorov, S., \& Vassilev, P. (2007). On an intuitionistic fuzzy approach for decision-making in medicine (Part 2), Int. J. Bioautomation, 7, 64-69.

[23] Yager, R. R. (2013). Pythagorean fuzzy subsets, In: Proceedings of the Joint IFSAWorld Congress and NAFIPS Annual Meeting, 57-61.

[24] Zadeh, L. A. (1965) Fuzzy sets, Inform. Control, 8, 338-353. 